\title{
METHODS FOR INCREASING OF BEAM INTENSITY IN UNDULATOR LINEAR ACCELERATOR*
}

\author{
E.S.Masunov, Moscow Engineering Physics Institute, Moscow, 115409 Russia.
}

The paper describes theoretical problems of ion focusing and acceleration in the high-intensity undulator linear accelerator (UNDULAC), where the accelerating force is produced by a combination of a radiofrequency field and undulator field. Both electrostatic and magnetostatic undulators are studied. The main factor limiting the beam intensity in the ion accelerator is space-charge forces. Three methods for increasing of the ion beam intensity in UNDULAC are discussed: (i) enlarging of the beam crosssection; (ii) acceleration of several beams in one RF structure; (iii) compensation of the space charge by acceleration of ions with different signs of the charge in the same bunch.

\section{INTRODUCTION}

Intensive particle beams with energy of $1-3 \mathrm{MeV}$ are used in neutron generators and for external injection of neutrals in nuclear fusion reactors of TOKAMAK type. For these purposes, beams with high average current and very small emittance growth are required. For energy $\mathrm{W}>1 \mathrm{MeV}$ linear RF accelerator instead of the accelerator of Cockroft Walton Cascade type must be applied.

Now, two principles of focusing and acceleration of low energy ions in resonant linear accelerators are well-known: 1) radio-frequency quadrupoles focusing (RFQ) and 2) alternating phase focusing (APF).

In UNDULAC there are no drift tubes .As a result, it is possible to use the methods for increasing of beam intensity, which can not be realized in RFQ and APF structures.

\section{PARTICLE MOTION EQUATIONS}

The motion equation of particle may be written, using Lagrange function

$$
\frac{d}{d t} \mathbf{P}=e \nabla(\mathbf{v} \cdot \mathbf{A}-\Phi)
$$

Where $\mathbf{P}=\mathbf{p}+e \mathbf{A}$ is the canonical momentum of the particle, $\mathbf{A}=\mathbf{A}_{v}+\mathbf{A}_{0}$ the overall vector potential of the RFfield $\mathbf{A}_{v}$ and the periodical magnetostatic field $\mathbf{A}_{0}$, $\Phi=\Phi_{v}+\Phi_{0}$ the overall electrostatic potential of the external field $\Phi_{v}$ and periodical electrostatic field of the undulator $\Phi_{0}$.

First of all, let us discuss the motion equation for a

\footnotetext{
* The research described in this publication was made possible in part by Grants \# MFQ 300 from the International Science Foundation and Russian Government.
}

particle in the magnetostatic undulator (UNDULAC-M) in the case when $\Phi=0$ and RF field harmonics are unsynchronized with the beam. The trajectories of the particles can be represented as a combination of fast oscillations $\tilde{\mathbf{r}}$ and slow variation $\mathbf{R}_{\mathrm{c}}$. The kinetic momentum of particle is represented by the sum of slowly varying and fast oscillating components $\mathbf{p}=\mathbf{p}_{c}+\tilde{\mathbf{p}}$. By averaging over fast oscillations, from (1) we obtain the equation, that describes the slow evolution $\mathbf{R}_{\mathrm{c}}$

$$
\frac{d^{2}}{d t^{2}} \mathbf{R}_{c}=-\frac{e^{2}}{2 m} \nabla\left\langle\left(\mathbf{A}_{v}+\mathbf{A}_{0}\right)^{2}\right\rangle \text {. }
$$

Taking into account only the main space harmonic of the magnetostatic undulator and TEM- or TE- wave in the waveguide structure, the equation (2) can be rewritten in the form

$$
\frac{d^{2}}{d t^{2}} \mathbf{R}_{c}=-\frac{\lambda}{8 \pi} \nabla U_{b}
$$

Where the potential function

$$
U_{b}=\mathbf{b}_{v}^{2}+\mathbf{b}_{0}^{2}-2 \mathbf{b}_{v} \cdot \mathbf{b}_{0} \sin \psi .
$$

Here $\mathbf{b}_{v, 0}=e \mathbf{B}_{v, 0}^{\perp} \lambda_{v, 0} /(2 \pi m c)$ the dimensionless amplitudes of the transverse components of the wave magnetic field $\mathbf{B}_{v}$ and undulator field $\mathbf{B}_{0} ; \mathbf{B}_{v, 0}=\operatorname{rot} \mathbf{A}_{v, 0} ; \psi=\omega \int_{0}^{z} d z_{1} / v_{s}-\tau+\psi_{0}$ - the particle phase in the combined wave field; $\omega$ - the frequency of RF field, $\tau=\omega t, \psi_{0}-$ the initial phase; $v_{s}=\lambda_{0} / \lambda_{v}-$ the normalized velocity of the synchronized particle, $\lambda_{v}$ - the RF-field wavelength, $\lambda_{0}$ - the undulator period.

The same equation can be obtained for the electrostatic undulator (UNDULAC-E) when $\mathbf{A}_{0}=0$

$$
\frac{d^{2}}{d t^{2}} \mathbf{R}_{c}=-\frac{\lambda}{8 \pi} \nabla U_{e}
$$

where the potential function

$$
U_{e}=\mathbf{e}_{v}^{2}+\mathbf{e}_{0}^{2}-2 \mathbf{e}_{v} \cdot \mathbf{e}_{0} \sin \psi .
$$

Here $\quad \mathbf{e}_{v, 0}=e \mathbf{E}_{v, 0} \lambda_{v, 0} /\left(2 \pi m c^{2}\right)$ - the dimensionless amplitudes of the basic RF-field harmonic $\mathbf{E}_{v}$ and the first electrostatic field harmonic $\mathbf{E}_{0}$. 
The acceleration gradient is proportional to the amplitudes of the RF and the undulator fields

$$
\frac{\Delta W}{\Delta z} \sim\left\{\begin{array}{l}
\mathbf{b}_{v} \cdot \mathbf{b}_{0} \\
\mathbf{e}_{v} \cdot \mathbf{e}_{0}
\end{array}\right\} \cos \psi
$$

The energy increase $\Delta \mathrm{W}$ on the length $\lambda_{0}$ is maximum, when $\mathbf{B}_{v}^{\perp} \| \mathbf{B}_{0}^{\perp}$ or $\mathbf{E}_{v} \| \mathbf{E}_{0}$. Therefore the choice of the magnetic (electrostatic) undulator type and its orientation depends on the RF-structure type [1].

Efficient capture and bunching of the beam can be obtained by the adiabatic growth of the values of $\mathbf{b}_{v, 0}$ and $\mathbf{e}_{v, 0}$ along the longitudinal axis and the corresponding increase of the undulator period $\lambda_{0}$ to maintain the beam synchronism with the combined wave field.

The choice of the functions $\mathbf{b}_{v}, \mathbf{e}_{v}$ and $\mathbf{b}_{0}, \mathbf{e}_{0}$ is not arbitrary because simultaneously with acceleration it is necessary to keep up the transverse focusing of the beam. In absence of the undulator, the transverse RF field can both focus and defocus the beam. The undulator without RF field can focus the beam. The combined field of the wave and the undulator accelerates particles in the longitudinal direction but defocusses them in the transverse direction.The total effect can be found only from the analysis of (4) and (4a). Equilibrium trajectories may exist for all particles of the beam, if two conditions are valid

$$
\nabla_{\perp}\left\{\begin{array}{l}
\mathbf{b}_{v}^{2}+\mathbf{b}_{0}^{2} \\
\mathbf{e}_{v}^{2}+\mathbf{e}_{0}^{2}
\end{array}\right\}=0, \quad \nabla_{\perp}\left\{\begin{array}{l}
\mathbf{b}_{v} \cdot \mathbf{b}_{0} \\
\mathbf{e}_{v} \cdot \mathbf{e}_{0}
\end{array}\right\}=0 .
$$

The motion around an equilibrium trajectory is stable if the potential function $U_{\mathrm{b}, \mathrm{e}}$ has an absolute minimum. These conditions obtained have to be tested considering coupling resonances. It is important to study non-linear oscillations of the beam particles for specification of the transverse focusing conditions.

In a simple case the potential function $U_{\mathrm{b}, \mathrm{e}}$ has been found without considering higher harmonics of the RF field and undulator field. It is important to avoid beam flaking when the beam interacts with the higher harmonics. Limitations imposed on the values of these harmonics specify the shape of the undulator poles, as well as the geometry of the electrodes that form the RF field.

\section{THE RIBBON BEAM IN THE UNDULATOR}

The current in the UNDULAC may be increased for the large cross-section beam. In the UNDULAC there is no drift tubes and the ribbon or hollow beams can be accelerated. Study of ribbon ion beam interaction with the RF-field in the plane electrostatic undulator was carried out in the papers [2-3]. Here the electrostatic undulator is combined with the RF system forming the transverse electric field.The required field distribution is provided by a system of transverse electrodes mounted in the resonator and dc-isolated between each other. The periodic undulator field is provided by electrostatic potential, which is supplied across the adjacent pairs of electrodes.Simultaneously, RF potentials are delivered to the electrodes. So the same electrodes are used to generate both fields.

This accelerator (UNDULAC-E) is suitable for demonstration of capabilities of this new method. The condition for providing efficient capture, bunching and acceleration of ions were found in [2]. The influence of coupling resonances on the choice of the field amplitudes was analyzed. An effect of higher harmonics of the space field on the beam dynamics was investigated. Some methods of formation of the required fields configuration were described in [4]. Computer codes for description of the ribbon ion beam dynamics in real fields were developed and the respective calculations were performed. The simulation results were in good agreement with theoretical estimations. Design of the RF-accelerating structure that showed a possibility to create the UNDULAC-E with a plane electrostatic undulator was proposed and studied experimentally [3].

The electrostatic version of UNDULAC is preferable at small values of the injection energy $(\mathrm{W}=30 \mathrm{KeV}$ for the proton beam). In the case of high injection energy (W> 100 $\mathrm{KeV}$ for the proton beam) we can substitute the magnetostatic undulator for the electrostatic one. For UNDULAC-M, it is impossible to obtain the large crosssection area of the beam because of technical problems connected with formation of strong magnetic fields in large volumes occupied by the beam. However, there is an opportunity to accelerate more then one beam in the magnetic channel.

\section{SEVERAL BEAMS IN THE UNDULATOR}

It is easy to accelerate the several beams in one RF structure of UNDULAC because there are no drift tubes. For UNDULAC-E, two (or N) ribbon beam could be accelerated if three (or $\mathrm{N}+1$ ) rows of electrodes are used. For UNDULAC-M, there is too an opportunity to accelerate more then one beam in the magnetic channel. The task is to choose a special symmetry of the transverse radio-frequency and periodical magnetostatic fields. The RF system must be a small transverse size to be located inside the undulator. Therefore, it is preferable to use a shielded multielectrode line where transverse electromagnetic (TEM) waves travel. The geometrical size of RF structure and the magnetic undulator must be found to maintain several equilibrium trajectories simultaneously. If the axis of the magnetic undulator coincides with that of RF system and the beam is injected along it, equations (6) are validated automatically. If the later condition is not valid, an equilibrium trajectory exists only if the field amplitudes and their transverse gradients are connected by the relations

$$
B_{0}=\frac{\lambda_{v}}{\lambda_{0}} B_{v}, \quad \nabla_{\perp} B_{0}=-\frac{\lambda_{v}}{\lambda_{0}} \nabla_{\perp} B_{v} .
$$


This result may be used at a high value of the aperture of the accelerating channel because it allows to inject particles beyond the axis and to place the beam closer to the poles of the undulators, in order to increase the acceleration gradient.

The simple example of UNDULAC-M with shieldedpair electrodes was considered in the paper [6]. In the paper [6] it was shown that for an axially symmetric undulator and two electrodes of line the potential function (4) has two absolute minima. So, the focusing conditions are valid for two beams. The choice of optimum parameters for bunching and acceleration of two ion beams might be satisfied similarly to the case of one beam.

Acceleration of more than two beams is possible if more than two longitudinal electrodes are used. For $\mathrm{N}$ electrodes in the axially symmetric undulator it is possible to accelerate $\mathrm{N}$ beams, while in the plane magnetic undulator $\mathrm{N}-1$ beams.

\section{THE SPACE CHARGE COMPENSATION}

Study of the possibility of simultaneous acceleration of both positive and negative ions with the identicial charge-tomass ratio in linear accelerators is great interest. The current limit of the ion beam can be substantially increased by using the space-charge compensation of positively $\mathrm{H}^{+}\left(\mathrm{D}^{+}\right)$and negatively $\mathrm{H}^{-} \quad\left(\mathrm{D}^{-}\right)$charged ions being accelerated simultaneously.

Let us consider the motion of positive and negative ions for a definite charge-to-mass ratio in UNDULAC. All the harmonics of RF field and the undulator field in the UNDULAC are asynchronous to the beam. After averaging over fast oscillations one can derive an expression for the effective potential function (4), (4a) that describes the averaged particle motion (3), (3a). The potential function $U_{\mathrm{b}, \mathrm{e}}$ depends on the particle charge squared, i.e. averaged motions of positive and negative charged ions are identical. It is important to note that both the main and high space harmonics have simular averaged influence on motion of the positive and negative ions. These ions will be within the same separatrix.

The averaged transverse trajectories $\mathbf{R}_{+,-}$of positively and negatively charged ions and their focusing conditions coincide. Nevertheless, fast transverse oscillations $\hat{\mathbf{r}}_{+,-}$of these ions occur in anti-phase $\left(\hat{\mathbf{r}}_{+}=-\hat{\mathbf{r}}_{-}\right)$.

Usually, amplitudes of fast oscillations are comparable with the mean beam size. For the space-charge compensation it is important that the transverse separation of the oppositely charged beams will not be large. If such the beams with the equal current $\left|I_{+}\right|=\left|I_{-}\right|$and the same initial emittances and velocities are injected into the accelerating channel, it is necessary at least that the difference between the mass center positions of the two beams is less then by $2 \tilde{r}$.

The result of mutual space-charge compensation depends primarily on behaviour of mass centers of the beams. Coherent transverse stability of a two-component ion beam was studing using the dispersion equation. The analysis of dispersion equation for this case showed that dipole resonances for two-component beam are not observed in presence of external focusing.

Computer simulation of a two-component ion beam, consisting of $\mathrm{H}^{+}$and $\mathrm{H}^{-}$was carried out by a macroparticle method in the paper [7]. The space-charge field was calculated from the 3D Poisson equation. The results of the numerial simulation of intense $\mathrm{H}^{+}$and $\mathrm{H}^{-}$beam dynamics confirm the analytical estimations.

\section{CONCLUSION}

Theoretical and experimental studies of the UNDULAC showed a possibility to create a new type of ion linear accelerator. In this accelerator there is no need in drift tubes. As a result, it is possible not only to enlarge the beam crosssection, but also to accelerate several beams in one channel and to use the space charge compensation. Many examples of realization of the $\mathrm{RF}$ and the undulator fields can be suggested for increasing of the beam intensity. Such ion accelerators can be used for the neutron generators and the nuclear fusion reactor.

\section{REFERENCES}

[1] E.S. Masunov, "Particle dynamics in a linear undulator accelerator", Zh.Tekn.Fiz,vol.60,\#9, pp.152157,1990 (in Russian).

[2] E.S.Masunov, A.P.Novikov, "Application of electrostatic undulators for acceleration of intense ion beams", Conf. Record of the 1991 Particle Accelerator Conference, San Francisco, California, May 1991, V.5, pp.3177-3179.

[3] E.S. Masunov, N.V. Leonov et.al., "A project of ion linear undulator accelerator with transverse RF-field", in the Third European Particle Accelerator Conference Proceedings, Berlin, March 1992, V. 1, pp. 572-574.

[4] E.S. Masunov, and A.P. Novicov, "Calculation of electrostatic and RF fields in UNDULAC-E with plane electrostatic undulator" Proc. of the Fourth European Particle Accelerator Conference, London, June 1994, V.2 , pp.12801282.

[5] E.S.Masunov, "Acceleration and Transverse Focusing of Ion Beams in Lineondutron." Proc. of the 1993 Particle Accelerator Conference, Washington, D.C., May 1993, V.3, pp.1681-1682.

[6] E.S. Masunov, "Acceleration of Two Ion Beams in Undulator Linear Accelerator" Proc. of the Fourth European Particle Accelerator Conference, London, June 1994, V.1, pp.820-822.

[7] E.S. Masunov, A.P. Novicov, "Spase-Charge Compensation Effects under Acceleration of Oppositely Charged Ion Bunchesin UNDULAC-E", Proc. of the Fourth European Particle Accelerator Conference, London, June 1994, V.2, pp.1171-1173. 